\title{
Gamma-Ray Constraint on Galactic Positron Production by MeV Dark Matter
}

\author{
John F. Beacom, ${ }^{1,2}$ Nicole F. Bell, ${ }^{3,4}$ and Gianfranco Bertone ${ }^{3}$ \\ ${ }^{1}$ Department of Physics, The Ohio State University, Columbus, Ohio 43210, USA \\ ${ }^{2}$ Department of Astronomy, The Ohio State University, Columbus, Ohio 43210, USA \\ ${ }^{3}$ NASA/Fermilab Astrophysics Center, Fermi National Accelerator Laboratory, Batavia, Illinois 60510-0500, USA \\ ${ }^{4}$ Kellogg Radiation Laboratory, California Institute of Technology, Pasadena, California 91125, USA
}

(Received 16 September 2004; published 5 May 2005)

\begin{abstract}
The Galactic positrons, as observed by their annihilation gamma-ray line at $0.511 \mathrm{MeV}$, are difficult to account for with astrophysical sources. It has been proposed that they are produced instead by dark matter annihilation or decay in the inner Galactic halo. To avoid other constraints, these processes are required to occur "invisibly," such that the eventual positron annihilation is the only detectable signal. However, electromagnetic radiative corrections to these processes inevitably produce real gamma rays ("internal bremsstrahlung"); this emission violates COMPTEL and EGRET constraints unless the dark matter mass is less than about $20 \mathrm{MeV}$.
\end{abstract}

DOI: 10.1103/PhysRevLett.94.171301

PACS numbers: 95.35.+d, 98.70.Rz, 98.70.Sa

Introduction.-The SPI camera on the INTEGRAL satellite has recently observed the $0.511 \mathrm{MeV}$ gamma-ray emission line arising from positron annihilation in the Galaxy [1-3]. The flux from the Galactic center region is $\Phi_{511}=\left(9.9_{-2.1}^{+4.7}\right) \times 10^{-4}$ photons $\mathrm{cm}^{-2} \mathrm{~s}^{-1}, \quad$ confirming earlier measurements [4-6]. The INTEGRAL data also provide new clues on the morphology of the emission region, which is consistent with a two-dimensional Gaussian of full width at half maximum (FWHM) of $9^{\circ}$, with a $2-\sigma$ uncertainty range covering $6-18^{\circ}$.

A variety of astrophysical sources of positrons have been proposed, among them compact objects, massive stars, supernovae, gamma-ray bursts, and cosmic rays (see, e.g., Refs. [7-11]). However, these astrophysical sources have difficulty accounting for the intensity of the positron annihilation flux, and especially for the morphology of the emission region, which so far in the INTEGRAL data shows neither a disk component nor discrete sources. These facts motivate consideration of an exotic mechanism for the positron production, and one associated with the dark matter concentration at the Galactic center is naturally suggested.

However, for dark matter candidates in the usually considered range of masses, $10 \mathrm{GeV}-10 \mathrm{TeV}$, the production of positrons by dark matter annihilation would be accompanied by the production of other kinematically allowed particles (for a recent review of dark matter candidates, see Ref. [12]). Even if direct production of gamma rays were suppressed, there would be a gamma-ray flux arising from the decays of secondaries (e.g., $\pi^{0}$, which are created in the hadronization of quarks). For annihilation of typical dark matter candidates to account for the observed positrons, the associated flux of high-energy gamma rays in the direction of the Galactic center would exceed the EGRET data by several orders of magnitude.

Boehm et al. [13] recently proposed that all of the characteristics of the observed signal could be well fit by a scenario in which light $(1-100 \mathrm{MeV})$ dark matter parti- cles annihilate only into $e^{+} e^{-}$pairs; the rate is controlled by the dark matter annihilation cross section (and its velocity dependence), and the morphology by the assumed dark matter density profile [13-15]. Since the mass is below $100 \mathrm{MeV}$, the only other kinematically allowed annihilations would be to gamma rays and neutrinos. To avoid the direct gamma-ray constraints (among them the cosmic gamma-ray background [16]) and to account for the required positron production rates, these modes are postulated to not occur. While the assumed dark matter mass is quite low, it is claimed that this model is consistent with all laboratory data $[13-15,17,18]$. In addition, the model is apparently consistent with astrophysical data and big-bang nucleosynthesis, depending on the assumptions made about the masses and couplings [19].

In the model of Boehm et al. [13], the positrons have a seemingly "invisible" birth, in that the dark matter annihilation produces only $e^{+} e^{-}$pairs. Another clever aspect of this model is that positrons below $100 \mathrm{MeV}$ will lose energy dominantly by ionization (avoiding production of possibly detectable inverse Compton and synchrotron radiation [20]) and will remain confined to the Galactic center region (required to reproduce the observed emission region). The positrons thus remain invisible until their annihilation into $0.511 \mathrm{MeV}$ gamma rays. Very similar considerations apply to models of dark matter decay which produce the observed positrons [21,22].

However, the dark matter annihilation process $\chi \chi \rightarrow$ $e^{+} e^{-}$is necessarily accompanied by the process $\chi \chi \rightarrow$ $e^{+} e^{-} \gamma$, arising from electromagnetic radiative corrections. This real gamma-ray emission, illustrated in Fig. 1, is known as internal bremsstrahlung, the name indicating that it arises from the Feynman diagram itself and is not due to propagation in a medium. In addition, the flux and spectrum of the internal bremsstrahlung gamma rays can be calculated with adequate accuracy without knowing the new particle physics which mediates the dark matter annihilation. The internal bremsstrahlung gamma rays reveal 


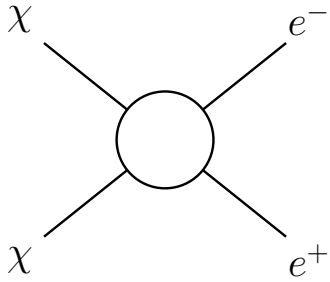

(a)

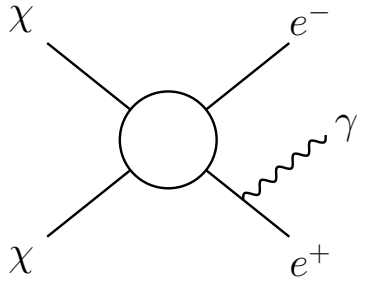

(b)
FIG. 1. (a) The dark matter annihilation channel $\chi \chi \rightarrow e^{+} e^{-}$ assumed by Boehm et al. [13]; the positron production and propagation occur invisibly, with only the eventual positron annihilation being detectable. The open circle represents unspecified new physics. (b) The observation of this Letter that positron production must be accompanied by detectable internal bremsstrahlung (a similar diagram with radiation from the electron is not shown).

the otherwise invisible dark matter annihilations, directly testing the central assumption of the proposed models, which is that the observed positrons were originally produced with energies up to $100 \mathrm{MeV}$. Astrophysical models would have to produce the same number of positrons, but at lower energies, typically a few $\mathrm{MeV}$.

We show that for most of the proposed dark matter mass range, the flux of internal bremsstrahlung gamma rays would be inconsistent with COMPTEL and EGRET measurements of diffuse radiation from the Galactic center region, thus requiring the dark matter mass to be less than about $20 \mathrm{MeV}$. This result is almost completely independent of assumptions about the physical conditions at the Galactic center or the new particle physics which mediates the dark matter annihilation. In fact, it is more general than just dark matter annihilation or decay, and prohibits any model for the positron production in which the positrons are created at energies above about $20 \mathrm{MeV}$. We also show that future Galactic gamma-ray data will be able to significantly improve the sensitivity of our constraint.

Internal bremsstrahlung. - The tree-level annihilation process $\chi \chi \rightarrow e^{+} e^{-}$is subject to radiative corrections, which affect the total cross section at $\mathcal{O}(\alpha)$, where $\alpha=$ $1 / 137$ is the fine-structure constant. These corrections are expected to be model dependent but small. The portion of the radiative corrections that governs the emission of real gamma rays, i.e., the finite part of the outer radiative corrections, is also expected to be small, but is process independent and factorizes from the tree-level cross section [23]. Generally, those results apply to soft gamma-ray radiation, but we show that they are adequately accurate for our purposes, even for large gamma-ray energies. Internal bremsstrahlung has been observed in a very wide variety of processes (one with similar positron energies is muon decay [24]).

The process $\chi \chi \rightarrow e^{+} e^{-}$from $\chi$ nearly at rest (typical halo velocities are $10^{-3} c$ ) produces monoenergetic electrons and positrons, with energies equal to the dark matter mass $m_{\chi}$. But when the gamma-ray energy is comparably large, it shares the phase space available to the final state. In order to reduce the process dependence, we use the finalstate internal bremsstrahlung probability from the kinematically similar process $e^{+} e^{-} \rightarrow \mu^{+} \mu^{-}[25,26]$, modified by replacing the muon mass with the electron mass (since the initial state is $\chi \chi$, it is appropriate to make this substitution while ignoring the identical-particle issue in $e^{+} e^{-} \rightarrow e^{+} e^{-}$).

The internal bremsstrahlung cross section is then

$$
\frac{d \sigma_{\mathrm{Br}}}{d E}=\sigma_{\mathrm{tot}} \times \frac{\alpha}{\pi} \frac{1}{E}\left[\ln \left(\frac{s^{\prime}}{m_{e}^{2}}\right)-1\right]\left[1+\left(\frac{s^{\prime}}{s}\right)^{2}\right],
$$

where $E$ is the gamma-ray energy, and $\sigma_{\text {tot }}$ the tree-level cross section, which factors out, an essential point. Here $s=4 m_{\chi}^{2}$ and $s^{\prime}=4 m_{\chi}\left(m_{\chi}-E\right)$. When $m_{\chi} \gg E, m_{e}$,

$$
\frac{d \sigma_{\mathrm{Br}}}{d E} \simeq \sigma_{\mathrm{tot}} \times \frac{4 \alpha}{\pi} \frac{\ln \left(2 m_{\chi} / m_{e}\right)}{E},
$$

which displays the familiar scaling factors. The internal bremsstrahlung probability is very small for the gammaray energy range which we consider, which is well above the soft singularity. We find the most stringent constraints when $E$ is large, near $m_{\chi}$, so we need to account for the phase space corrections included in Eq. (1) but not the simpler Eq. (2).

The flux of internal bremsstrahlung gamma rays is proportional to the positron production rate, which is determined from the $0.511 \mathrm{MeV}$ intensity. Positron annihilation occurs either via direct annihilation into two $0.511 \mathrm{MeV}$ gamma rays or via the formation of a positronium bound state. A singlet state (parapositronium), which decays to two $0.511 \mathrm{MeV}$ gamma rays, is formed $25 \%$ of the time, while the triplet state (orthopositronium), which decays to three continuum gamma rays, is formed $75 \%$ of the time. The ratio of direct versus positronium annihilation can be measured by comparing the $0.511 \mathrm{MeV}$ line intensity to the continuum intensity. It is customary to define the positronium fraction [27] as

$$
f=\frac{2}{1.5+2.25\left(\Phi_{511} / \Phi_{\text {cont }}\right)} .
$$

This fraction depends on the physical conditions of the gas at the Galactic center, and observations suggest that $f=$ $0.93 \pm 0.04$, implying that most positrons annihilate via positronium [28]. This means that the dark matter annihilation rate and hence the internal bremsstrahlung rate are about 4 times larger than the positron annihilation rate observed via the $0.511 \mathrm{MeV}$ line.

The spectrum of internal bremsstrahlung gamma rays per $0.511 \mathrm{MeV}$ gamma ray is therefore

$$
\frac{d N_{\mathrm{Br}}}{d E}=\left[\frac{f}{4}+(1-f)\right]^{-1} \times \frac{1}{2} \times \frac{1}{\sigma_{\mathrm{tot}}} \frac{d \sigma_{\mathrm{Br}}}{d E},
$$

where we have accounted for the fraction of positrons that 
annihilate into $0.511 \mathrm{MeV}$ gamma rays, and that each annihilation produces two $0.511 \mathrm{MeV}$ gamma rays.

Three key assumptions. - We now identify three key assumptions of the model of Boehm et al. [13]; violations of these assumptions act in the sense of strengthening the internal bremsstrahlung constraint.

First, it is assumed that the positron diffusion length is small compared to the size of the Galactic center region (this may not be the case; see Ref. [11]). Then the spatial distributions of dark matter annihilation and the internal bremsstrahlung gamma rays would be the same as the observed spatial distribution of $e^{+} e^{-}$annihilation. If this assumption were violated, then the flux per steradian of internal bremsstrahlung gamma rays would be larger (and would come from a smaller angular region).

Second, it is assumed that the positrons are brought to rest by ionization losses quickly, so that the rates of dark matter annihilation and positron annihilation are in equilibrium. If this assumption were violated, then the positron annihilation rate today would reflect the dark matter annihilation rate in the past, while the internal bremsstrahlung rate would reflect the (larger) dark matter annihilation rate now (continued gravitational collapse makes dark matter halos increasingly dense).

Third, it is assumed that the positrons annihilate at rest. If this assumption were violated, then, to produce the same $0.511 \mathrm{MeV}$ flux, the dark matter annihilation and internal bremsstrahlung gamma-ray rates would have to be larger. Additionally, positron annihilation in flight could produce a detectable high-energy gamma-ray signature of their own, though it would depend on the details of the physical conditions in the Galactic center.

COMPTEL/EGRET constraints. - The flux of internal bremsstrahlung gamma rays per steradian is

$$
\frac{d \Phi_{\mathrm{Br}}}{d E} \simeq \frac{1}{2} \Phi_{511} \frac{d N_{\mathrm{Br}}}{d E} \frac{1}{\Delta \Omega},
$$

where we have assumed that $1 / 2$ of the $0.511 \mathrm{MeV}$ flux is emitted from an angular region $\Delta \Omega$ given by the Gaussian FWHM of $9^{\circ}$. This becomes

$$
\frac{d \Phi_{\mathrm{Br}}}{d E} \simeq\left(4.2_{-0.9}^{+2.0}\right) \times 10^{-2} \frac{1}{\sigma_{\mathrm{tot}}} \frac{d \sigma_{\mathrm{Br}}}{d E} \mathrm{~cm}^{-2} \mathrm{~s}^{-1} \mathrm{sr}^{-1} .
$$

The corresponding internal bremsstrahlung gamma-ray spectra are shown in Fig. 2. The energy dependence of the cross section in Eq. (1) gives spectra which peak at $E \simeq$ $m_{\chi}$, and fall off as $1 / E$; for easier comparison with the data, we plot $E^{2}$ times the spectra. The offsets between the solid lines at low $E$ reflect just the $\ln \left(2 m_{\chi} / m_{e}\right)$ factor in the approximate and process-independent Eq. (2). The mild process dependence [the difference between Eq. (1) and (2) in the treatment of the phase space] is seen in Fig. 2 in the slight turnovers in the solid lines before the end points, as well as a general reduction by a factor $\lesssim 2$. Had we used just Eq. (2), our constraints would have been somewhat too strong.

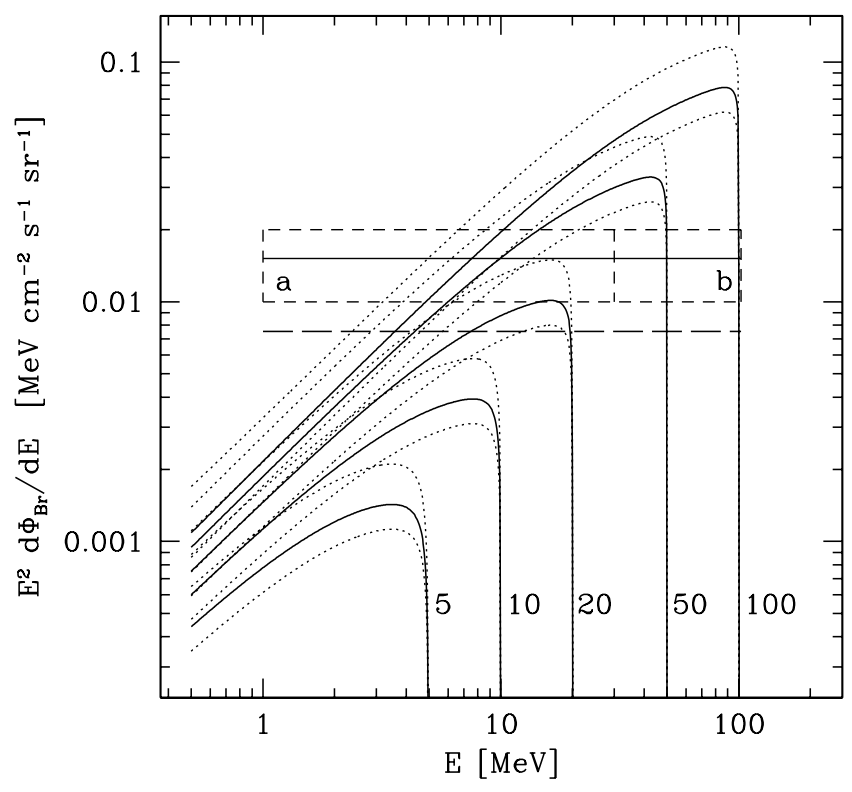

FIG. 2. The solid lines show the internal bremsstrahlung spectra labeled by the assumed dark matter masses in $\mathrm{MeV}$, and normalized to produce the observed Galactic $0.511 \mathrm{MeV}$ flux (with the light dotted lines corresponding to its uncertainty); we assumed that the FWHM of the $0.511 \mathrm{MeV}$ emission region is $9^{\circ}$. The approximate (a) COMPTEL and (b) EGRET data and their uncertainty band are shown by the box, and the long-dashed line below it indicates the rough constraint on the maximum allowed contribution of internal bremsstrahlung gamma rays.

We can constrain the internal bremsstrahlung contribution by comparing to COMPTEL and EGRET measurements of the diffuse gamma-ray flux from the Galactic center region [29-31]. Those fluxes were averaged over a Galactic center region of $\pm 5^{\circ}$ in latitude and $\pm 30^{\circ}$ in longitude, but were shown to have a mild variation across that region. The observed $0.511 \mathrm{MeV}$ emission is from a smaller region of FWHM $9^{\circ}$. Thus, in order to constrain an internal bremsstrahlung contribution to the central circle, we must first subtract the diffuse astrophysical contribution as measured over the whole COMPTEL/EGRET rectangle. We have conservatively assumed that the COMPTEL/ EGRET data band is given at one sigma, and that an excess contribution of more than about $50 \%$ could not be tolerated. A fit to the full energy and angular dependence of the signal and background would yield a more stringent constraint.

Even with our very conservative treatment, we can easily constrain the dark matter mass in the Boehm et al. model [13] to $m_{\chi} \lesssim 20 \mathrm{MeV}$ (for decaying dark matter $[21,22]$ we determine a similar upper limit). The uncertainties on the COMPTEL/EGRET data (as well as our approximate handling thereof), the $0.511 \mathrm{MeV}$ flux, and the positronium fraction are relatively unimportant, though improvements would be welcomed. The largest uncertainty on the constraint arises from the uncertainty in the size of the $0.511 \mathrm{MeV}$ emission region. Following Refs. [1-3,13], we assumed $9^{\circ}$, but the $2-\sigma$ range spans $6^{\circ}-18^{\circ}$, allow- 
ing an order of magnitude in the internal bremsstrahlung flux per steradian; this corresponds to varying the upper limit on $m_{\chi}$ from 10 to $60 \mathrm{MeV}$. Improved results from INTEGRAL on the size of the emission region are thus eagerly awaited.

Discussion and conclusions. - In order to explain the Galactic positron excess, as well as the smooth and centrally symmetric morphology of the $0.511 \mathrm{MeV}$ emission observed by INTEGRAL [1-3], Boehm et al. [13] (see also Refs. [14,15,17-19]) proposed an intriguing model where $e^{+} e^{-}$pairs are produced by the annihilation of light (1$100 \mathrm{MeV}$ ) dark matter candidates, which apparently can evade all present accelerator and astrophysical constraints. We have pointed out the central assumption of this model, i.e., that energetic positrons may be produced in the Galactic center with no other observational consequences other than their eventual annihilation into $0.511 \mathrm{MeV}$ gamma rays. We have shown that such an invisible birth is prohibited by the emission of internal bremsstrahlung gamma rays from the original dark matter annihilations unless the dark matter mass is less than about $20 \mathrm{MeV}$, disallowing most of the proposed range. We have arrived at this constraint in a very conservative fashion, and expect that improved gamma-ray data and a more sophisticated analysis will significantly improve the sensitivity. Our constraint is very nearly independent of the new physics of the dark matter particles, and would be strengthened by relaxing the assumptions on the physical conditions at the Galactic center.

Although our results have been presented in the context of dark matter annihilation, they are much more general. Any steady-state and isotropic mechanism, whether based on standard astrophysics or requiring new particle physics, and which produces energetic positrons, will be accompanied by internal bremsstrahlung gamma rays, with the relative rate nearly independent of the tree-level cross section. Thus for any mechanism that creates enough positrons to account for the $0.511 \mathrm{MeV}$ line, if those positrons are produced above about $20 \mathrm{MeV}$, the accompanying internal bremsstrahlung will violate the COMPTEL/ EGRET constraints. Dark matter decay is a possible example [21,22]. The ultimate low-energy sensitivity of the technique will be reached when the dominant sources are astrophysical sites that produce gamma rays and positrons at comparable (tree-level) rates.

We are grateful to C. Boehm, S. Boggs, E. Braaten, V. Cirigliano, D. Hartmann, M. Leising, D. Rainwater, and M. Ramsey-Musolf for comments on the manuscript. J.F. B. was supported by funds from The Ohio State University, and N.F.B. and G. B. by Fermilab (operated by URA under DOE Contract No. DE-AC02-76CH03000) and by NASA Grant No. NAG5-10842.
[1] P. Jean et al., Astron. Astrophys. 407, L55 (2003).

[2] J. Knödlseder et al., astro-ph/0309442.

[3] G. Weidenspointner et al., astro-ph/0406178.

[4] W. N. Johnson and R. C. Haymes, Astrophys. J. 184, 103 (1973).

[5] M. Leventhal, C. J. MacCallum, and P.D. Stang, Astrophys. J. 225, L11 (1978).

[6] W. Purcell et al., Astrophys. J. 491, 725 (1997).

[7] K. Chan and R. Lingenfelter, Astrophys. J. 405, 614 (1993).

[8] P. A. Milne, J. D. Kurfess, R. L. Kinzer, and M. D. Leising, New Astron. Rev. 46, 553 (2002).

[9] C. D. Dermer and R. J. Murphy, astro-ph/0107216.

[10] M. Casse, B. Cordier, J. Paul, and S. Schanne, Astrophys. J. 602, L17 (2004).

[11] G. Bertone, A. Kusenko, S. Palomares-Ruiz, S. Pascoli, and D. Semikoz, astro-ph/0405005.

[12] G. Bertone, D. Hooper, and J. Silk, Phys. Rep. 405, 279 (2005).

[13] C. Boehm, D. Hooper, J. Silk, M. Casse, and J. Paul, Phys. Rev. Lett. 92, 101301 (2004).

[14] C. Boehm, P. Fayet, and J. Silk, Phys. Rev. D 69, 101302 (2004).

[15] D. Hooper, F. Ferrer, C. Boehm, J. Silk, J. Paul, N. W. Evans, and M. Casse, Phys. Rev. Lett. 93, 161302 (2004).

[16] P. J. Zhang and J. F. Beacom, Astrophys. J. 614, 37 (2004).

[17] C. Boehm, T. A. Ensslin, and J. Silk, J. Phys. G 30, 279 (2004).

[18] C. Boehm and P. Fayet, Nucl. Phys. B683, 219 (2004).

[19] P. D. Serpico and G. G. Raffelt, Phys. Rev. D 70, 043526 (2004).

[20] A. W. Strong and I. V. Moskalenko, Astrophys. J. 509, 212 (1998).

[21] C. Picciotto and M. Pospelov, Phys. Lett. B 605, 15 (2005).

[22] D. Hooper and L. T. Wang, Phys. Rev. D 70, 63506 (2004).

[23] M.E. Peskin and D. V. Schroeder, An Introduction to Quantum Field Theory (Addison-Wesley, Reading, 1995), Chap. 6.

[24] R. R. Crittenden, W. D. Walker, and J. Ballam, Phys. Rev. 121, 1823 (1961).

[25] H. U. Martyn, in Quantum Electrodynamics, edited by T. Kinoshita (World Scientific, Singapore, 1990).

[26] F. A. Berends and A. Bohm, in High Energy ElectronPositron Physics, edited by A. Ali and P. Soding (World Scientific, Singapore, 1998).

[27] B. L. Brown and M. Leventhal, Astrophys. J. 319, 637 (1987).

[28] R. L. Kinzer, P. A. Milne, J. D. Kurfess, M. S. Strickman, W. N. Johnson, and W. R. Purcell, Astrophys. J. 559, 282 (2001).

[29] A. W. Strong, H. Bloemen, R. Diehl, W. Hermsen, and V. Schoenfelder, astro-ph/9811211.

[30] A. W. Strong, I. V. Moskalenko, and O. Reimer, Astrophys. J. 537, 763 (2000); 541, 1109(E) (2000).

[31] A.W. Strong, I. V. Moskalenko, and O. Reimer, Astrophys. J. 613, 962 (2004). 